\title{
TRASCENDENCIA DE LA ENSEÑANZA DEL CUIDADO ENFERMERO Y SU REPERCUCION SOCIAL REGION SUR PERÚ 2012 - 2013
}

\section{Trascendence of the nursing care teaching and its social impact Región Sur Perú 2012 - 2013}

\author{
Sonia Velásquez Rondón $n^{1, a, b, c}$
}

\author{
${ }^{1}$ Facultad de Enfermería. Universidad Nacional San Agustín de Arequipa, Perú \\ a Lic.en Enfermería Mag.en Salud Pública.cDoctora en Salud Colectiva y en Enfermería
}

\section{RESUMEN}

Objetivo: Analizar la transferencia del cuidado enfermero como práctica social de los enfermeros de la macro región sur Arequipa 2014. Material y métodos: Tipo descriptivo, con diseño de correlación, la muestra fue de 720 enfermeras y 830 personas enfermas. Resultados: El 67\% de enfermeras en su formación solo ejecutaron procedimientos, un $77 \%$ no aplican el cuidado de enfermería, no conocían las teorías de enfermería, realizaron prácticas en hospitales en el $85 \%$. Un 92\% define parcialmente el cuidado enfermero no lo aplica el $8 \%$. El $87 \%$ de enfermos tuvieron experiencias negativas, trato inadecuado en el 76\%, no interactúan con ellos en el $80 \%$. Conclusiónes: La trascendencia de la formación del cuidado enfermero se relaciona con la repercusión en la sociedad $\left(X^{2}>0.01\right)$

Palabras claves: Enseñanza del cuidado enfermero, repercusión social

\section{SUMMARY}

Objective: To analyze the transfer of nursing care as a social practice of the nurses of the southern region of Arequipa 2014. Material and methods: Descriptive type, with correlation design, the sample was 720 nurses and 830 sick people. Results: $67 \%$ of nurses in their training only performed procedures, $77 \%$ did not apply nursing care, did not know nursing theories, did practice in hospitals in $85 \%$. A $92 \%$ partially defines the nursing care is not applied $8 \% .87 \%$ of patients had negative experiences, inadequate treatment in $76 \%$, did not interact with them in $80 \%$. Conclusions: The importance of nursing care is related to the repercussion in society $\left(x^{2}>0.01\right)$

Key words: Teaching nursing care, social repercussion

\section{INTRODUCCIÓN}

En los últimos años las instituciones de salud están saturadas por gran afluencia de personas enfermas con pronóstico grave y que son internados en los diversos servicios para mejorar su situación de salud estando a cargo de un equipo de salud eficiente que actúa en situaciones de gran tensión debido al estado crítico en que se encuentran los enfermos, a los que se debe dar cuidado de enfermería oportuno y con calidad para superar la situación que vive.
Las enfermeras son el único personal de salud que está a cargo de las personas enfermas durante las veinticuatro horas, son quienes satisfacen sus necesidades, valoran continuamente el estado de salud de los enfermos, cumplen las prescripciones dadas por el médico haciendo reportes constantes, esta función la cumplen con éxito, a ella dedican gran tiempo y esfuerzo; superado el periodo crítico continúan brindando cuidado a dichos enfermos, con calidez y calidad. Sin embargo, la actuación de la enfermera generalmente se orienta al cumplimiento de 
funciones interdependientes: aplicación de la indicación terapéutica, monitoreo de funciones, registro de la información entre otros, dejando de lado las funciones independientes que corresponden a la aplicación de su propia ciencia, cuidando a las personas en forma holística, tal como expresa Kerouac (1). "La enfermería tiene que tener en cuenta al enfermo en su contexto familiar, social y cultural para saber qué prefiere y acogerlo en esa experiencia única que vive, la cual le dejara sensaciones y emociones positivas y negativas.

Meleis (2) expresa "Aunque las enfermeras / os individualmente han cuidado y desarrollado el cuidado de pacientes en todos los rincones de la tierra, no creo que hayamos alcanzado plenamente nuestro potencial para producir un impacto colectivo sobre el cuidado de la salud y de la vida de las comunidades. La disciplina de enfermería y las enfermeras / os como fuente y recurso del conocimiento de enfermería pueden influir no solamente sobre el cuidado de los pacientes, sino también sobre las políticas que orientan el cuidado de la salud.

En los últimos años se ha analizado las actividades que realizan las enfermeras en los diferentes servicios de salud, las cuales solo son funciones delegadas, satisfaciendo las necesidades solo de la esfera física y biológica, dichas actividades son desarrolladas con seguridad, experticia y eficiencia, las cuales son señaladas como excelentes según su criterio, el cual es totalmente opuesto a lo expresado por los enfermos. Es decir hay un reduccionismo biológico del cuidado y una deshumanización de lo humano, lo que muestra que el cuidado de enfermería está fragmentado, que la participación de la persona enferma o del familiar está dirigida solo al aprendizaje de esta esfera, hay un quehacer solitario, ineficaz y deficiente. (Medina, L) (3).
En diversas oportunidades los enfermeros expresan que es imposible brindar cuidado de calidad porque no tienen el tiempo necesario, que la gravedad del enfermo le impide dar un cuidado holístico, porque su actuar es inmediato, rápido, cualquier demora pone en riesgo la vida de la persona enferma, dicha aseveración muestra que el enfermo se ha cosificado porque el cuidado es interactivo, es hablar, tocar, acariciar, mirar, es dar confianza aun en la inconsciencia, es hablar aun en el silencio, es dar sosiego y paz aun en la adversidad, es trascender aun en lo inminente de la muerte, en ese sentido no se practica el cuidado enfermero desde su ciencia, desde su abordaje profesional. (Waldow, V) (4).

La indagación genera algunas interrogantes: ¿Es que se le desconoce? ¿Solo se formó el Hacer y no el Ser? ¿Porque es tan complicado dar cuidado si es la esencia de la enfermería? ¿Cuál es la concepción que tienen las enfermeras para brindar cuidado? Todos estos cuestionamientos han sido plasmados en la investigación para poder clarificar la problemática descrita, asumiendo que hay la posibilidad de que exista deficiencia en la formación de la cuidadora.

Las experiencias de aprendizaje para el cuidado enfermero son fuente de análisis, involucra tomar decisiones junto a otra persona sana o enferma, que tiene su propio concepto de enfermedad, su forma de curar, su explicación de estar sana, por lo tanto la planificación del cuidado enfermero parte de dicha información, la cual puede estar soportada por una teoría, orientando el actuar de la enfermera, es en esa perspectiva que debiera fortalecerse la formación profesional, en consecuencia, las capacidades que adquieran las estudiantes además de la habilidad y destreza, es el reconocimiento de las características que cada caso clínico presenta, lo que fortalece su bagaje de experiencias alcanzadas en su formación profesional, sin embargo en la actualidad, la 
preocupación va dirigida a la realización de una serie de procedimientos los que deben ser en gran porcentaje porque de esa manera hay metas logradas, la evaluación también se orienta hacia este aspecto , a los trabajos, a realizar el proceso de enfermería. Así mismo se privilegia $y$ fortalece las actividades prácticas en los hospitales, dejando de lado a la comunidad, hay una formación orientada al daño y a la enfermedad, con currículos cuyo espacio de prácticas en un alto porcentaje se desarrolla en hospitales (Baez) (5). Este actuar que se da en todas las asignaturas, durante cinco años de formación, han producido un pensamiento reduccionista del enfermero, porque no puede adecuar luego su actuación en la comunidad, trasladando sus saberes y actuar a un espacio diferente lo que le genera malestar en su ejercicio profesional (Waldow, V) (4).

Poblete (6) expresa que con el avance de la tecnología y de la ciencia en su conjunto se ha producido grandes cambios y transformaciones, sin embargo la formación de las enfermeras no ha cambiado en los últimos tiempos, este hecho está repercutiendo en el cuidado que brinda la enfermera, observándose una atención despersonalizada, deshumanizada, desarticulada y desfasada de una realidad concreta, los mensajes de descontento que dan los usuarios hacen urgente un cambio en las entidades formadoras en cuanto a sus currículos para hacer que el cuidado enfermero trascienda la formación y la atención. (Almenares, 2007) (7). Al respecto Murrain ( 8 ), manifiesta que a pesar de ser el mayor grupo de fuerza laboral para dar cuidado no es visible su actividad.

Las repercusiones se presentan en lo cotidiano donde existe mal trato, deshumanización, cosificación, ineficiencia, deterioro moral y ético al considerar que el paciente cumple ese rol, solo esperar para recibir atención( Rivera) (9).
Baez, F ( 2011) (5) Colombia expresan que el cuidado de enfermería envuelve y comparte la experiencia humana a través de una relación transpersonal y de respeto, Bernal $\mathrm{M}$, Ponce G. en México 2009 (10), manifiestan que es fundamental que la enseñanza en Enfermería sea integral; activa; e integrativa; de tal forma que lleve al desarrollo de valores; conocimientos y relaciones sociales para formar seres humanos que cuiden a seres humanos Rivera, N (2007) (9), manifiesta que el cuidado es la esencia (eje y corazón) de la práctica de enfermería, que moviliza a la persona hacia la armonía entre cuerpo, mente y alma, a través de una relación de ayuda y confianza entre la persona cuidada y el cuidador, generando conocimiento, respeto y ayuda mutua, rescatando siempre la dignidad humana en la asistencia de enfermería, dentro de un marco de amor. Añade Jiménez año 2013 (11), que existe un impacto en la visión del cuidado y la persona, centrando la atención en la enfermedad, en lo biológico y la técnica, enfoque que ha predominado hasta fechas recientes, a diferencia de Muñoz (12), que dan significado al Cuidado, expresan que es dar atención integral al paciente, tener en cuenta al individuo como persona, proporcionando un completo bienestar físico, social y psicológico encaminado a satisfacer las necesidades humanas. Recientemente existe interés en comprender al hombre en su dimensión de individuo, en percibirlo como tal y trascender a la humanización del cuidado, para ello las teóricas han incursionado en otras áreas del conocimiento (Prieto, 2007) (13).

Existe por lo tanto la necesidad de mostrar las debilidades existentes en la formación, para plantear cambios en dicho proceso mejorando las bases filosóficas y epistemológicas del cuidado enfermero, cambiando esquemas y reconstruyendo la esencia de enfermería, su objeto de trabajo que es el cuidado, para mejorar la calidad del cuidado brindado satisfaciendo las necesidades de la población, propendiendo a un acercamiento hacia el ser cuidado, hacia su humanización la que debiera 
iniciarse desde la academia, transformando los saberes, caso contrario, se separa una dualidad existente desde el inicio de la enfermería: cuidadora - ser cuidado, porque ya no interpretamos su mensaje, su necesidad, su existencia.

\section{MATERIALES Y METODOS}

La investigación fue de tipo descriptivo con un diseño de correlación. La población en estudio estuvo constituida por 1200 enfermeras, y 1500 de personas enfermas. La muestra aplicando la fórmula de población finita fue de 720 enfermeras y 830 personas enfermas, el muestreo es aleatorio simple.

Para el desarrollo de la investigación se utilizó como método la encuesta, como técnica el cuestionario y como instrumento el formulario con 30 reactivos, se validaron con juicios de expertos y el alfa de Cronbach. La prueba estadística utilizada fue el chi cuadrado.

\section{RESULTADOS}

$58 \%$ de enfermeras tienen edades de 35 a 37 años, trabajan de 10 años a mas en el $48 \%$, proceden el $50 \%$ de zonas urbanas. Un $67 \%$ de la población en estudio refiere que su formación se basaba solo en ejecutar procedimientos. El $77 \%$ de enfermeras no aplican el cuidado de enfermería, no habiendo recibido información sobre las teorías de enfermería.

El $85 \%$ de enfermeras realizo sus prácticas en hospitales. El 92\% define parcialmente el cuidado enfermero y no lo aplica en su cotidiano en el $8 \%$. El $87 \%$ de personas

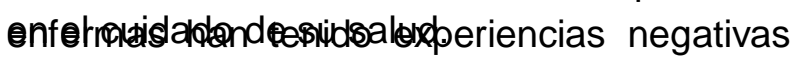

El $76 \%$ de enfermos indican que la enfermera tiene un trato inadecuado, el $80 \%$ indican que no interactúan con ellos.

La prueba estadística de chi cuadrado establece que la trascendencia de la formación del cuidado enfermero repercute negativamente en la sociedad.
Tabla 1. Población en estudio por enseñanza del cuidado enfermero Región Sur 2012 - 2013

\begin{tabular}{|c|c|c|}
\hline $\begin{array}{c}\text { Enseñanza del cuic ado } \\
\text { enfermero }\end{array}$ & $\mathbf{N}^{\circ}$ & $\%$ \\
\hline Si le dieron & 75 & 10,4 \\
\hline Parcialmente le dierc $n$ & 123 & 17,1 \\
\hline No le dieron & 522 & 72,5 \\
\hline Total & 720 & 100 \\
\hline
\end{tabular}

Tabla. 2. Población en estudio por enseñanza de modelos teorías en enfermería Región Sur 2012 - 2013

\begin{tabular}{crc}
\hline $\begin{array}{l}\text { Enseñanza de modelos } \\
\text { y teorías de enfermería }\end{array}$ & \multicolumn{1}{c}{$\mathbf{N}^{\circ}$} & $\%$ \\
\hline Si le enseñaron & 75 & 10,4 \\
No le enseñaron & 645 & 89,6 \\
\hline Total & $\mathbf{7 2 0}$ & $\mathbf{1 0 0}$ \\
\hline
\end{tabular}

Tabla 3. Población en estudio por centro de prácticas enfermería Región Sur 2012 - 2013

\begin{tabular}{lcc}
\hline Centro de practicas & $\mathbf{N}^{\circ}$ & $\%$ \\
\hline Solo Hospital & 612 & 85,0 \\
solo Centro periférico & 66 & 9,1 \\
Ambos & 43 & 5,9 \\
\hline \multicolumn{1}{c}{ Total } & $\mathbf{7 2 0}$ & $\mathbf{1 0 0}$ \\
\hline
\end{tabular}

Tabla. 4. Población en estudio por conceptualización del cuidado enfermero enfermería Región Sur 2012 - 2013

\begin{tabular}{lrr}
\hline $\begin{array}{c}\text { Concept. del Cuidado } \\
\text { Enfermero }\end{array}$ & \multicolumn{1}{c}{$\mathbf{N}^{\circ}$} & \multicolumn{1}{c}{$\%$} \\
\hline Totalmente & 14 & 1,9 \\
Parcialmente & 623 & 92,0 \\
No Conceptualiza & 83 & 11,5 \\
\hline \multicolumn{1}{c}{ Total } & $\mathbf{7 2 0}$ & $\mathbf{1 0 0}$ \\
\hline
\end{tabular}

Tabla. 5. Población en estudio por aplicación del cuidado de enfermería Región Sur 2012 - 2013

\begin{tabular}{lrr}
\hline $\begin{array}{c}\text { Aplicación del cuidado } \\
\text { enfermero }\end{array}$ & $\mathbf{N}^{\circ}$ & $\%$ \\
\hline Si Aplica & 14 & 1,9 \\
Parcialmente Aplica & 649 & 90,1 \\
No Aplica & 57 & 8,0 \\
\hline \multicolumn{1}{c}{ Total } & $\mathbf{7 2 0}$ & $\mathbf{1 0 0}$ \\
\hline
\end{tabular}

Tabla.6. Población en estudio por experiencias del cuidado enfermero Región Sur 2012 - 2013

\begin{tabular}{lrr}
\hline $\begin{array}{l}\text { Experiencias del } \\
\text { Cuidado Enfermero }\end{array}$ & \multicolumn{1}{c}{ No $^{\circ}$} & \multicolumn{1}{c}{$\%$} \\
\hline Positivas & 52 & 6,3 \\
Negativas & 722 & 87,0 \\
Neutro & 56 & 6,7 \\
\cline { 2 - 3 } Total & & $\mathbf{1 0 0}$
\end{tabular}

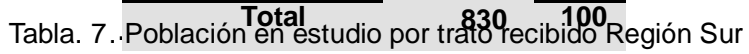

\begin{tabular}{ccc}
\multicolumn{3}{c}{$2012-2013$} \\
\hline Trato recibido & $\mathbf{N}^{\circ}$ & $\%$ \\
Mal trato & 630 & 76,0 \\
Buen trato & 200 & 24,0 \\
\hline Total & $\mathbf{8 3 0}$ & $\mathbf{1 0 0}$
\end{tabular}

Tabla. 8. Población en estudio por percepción del cuidado enfermero Región Sur 2012 - 2013

\begin{tabular}{ccc}
\hline $\begin{array}{c}\text { Percepción del } \\
\text { cuidado enfermero }\end{array}$ & $\mathbf{N}^{\circ}$ & $\%$ \\
\hline $\begin{array}{c}\text { Si, interactúa } \\
\text { No, interactúa }\end{array}$ & 166 & 20,0 \\
\hline Total & $\mathbf{8 3 0}$ & $\mathbf{1 0 0}$ \\
\hline
\end{tabular}


Tabla. 9. Trascendencia del cuidado enfermero y su repercusión social Región Sur 2012 - 2013

\begin{tabular}{lccccc}
\hline Trascendencia del & \multicolumn{3}{c}{ REPERCUSION SOCIAL } \\
$\quad$ Cuidado & \multicolumn{2}{c}{ Positiva } & \multicolumn{2}{c}{ Negativa } \\
Enfermero & & $\mathbf{N}^{\circ}$ & $\%$ & $\mathbf{N}^{\circ}$ & $\%$ \\
\cline { 5 - 6 } Buena & 3 & 0,3 & 20 & 2,4 \\
Parciamente buena & 52 & 6,2 & 690 & 81,1 \\
Mala & 6 & 0,7 & 59 & 7,1 \\
\hline Total & $\mathbf{6 1}$ & $\mathbf{7 , 3}$ & $\mathbf{7 6 9}$ & $\mathbf{9 2 , 6}$ \\
\hline \multicolumn{1}{c}{$\mathrm{X}^{2}>0.01$} & & & &
\end{tabular}

\section{DISCUSION}

El cuidado es el objeto de estudio de los enfermeros e implica satisfacer las necesidades de las personas en todas las esferas: biológico, social, psicológico, cultural y espiritual. El $92 \%$ de los enfermeros conceptualiza parcialmente el cuidado enfermero a semejanza de Bernal y Ponce (10) que manifiestan que es fundamental que la enseñanza en Enfermería sea integral; activa; e integrativa; de tal forma que lleve al desarrollo de valores; conocimientos y relaciones sociales para formar seres humanos que cuiden a seres humanos, de esta forma brindara un cuidado de calidad, corrobora Muñoz y colaboradores dando significado al Cuidado, expresan que es dar atención integral al paciente, tener en cuenta al individuo como persona, proporcionando un completo bienestar físico, social y psicológico encaminado a satisfacer las necesidades humanas. (12).

En el estudio muestra que el $92.1 \%$ de los enfermeros aplica parcialmente el cuidado enfermero

El $87 \%$ de las personas enfermas tuvieron una experiencia negativa de cuidado enfermero en igualdad a Báez y otros (5) que identifica que el cuidado es considerado como un hacer monótono, lineal y sin sentido, dentro de la racionalidad técnica y el enfoque biomédico, concordando con Kerouac,(1) indica que la enfermería tiene que tener en cuenta al enfermo en su contexto familiar, social y cultural para saber qué prefiere y acogerlo en esa experiencia única que vive, la cual le dejara sensaciones y emociones positivas y negativas.

El $76 \%$ de enfermos percibió mal trato a semejanza de Poblete (6) que indica que los nacientes valoran más los asnectos del cuidado que tienen relación con la comunicación, el afecto y la atención que se le presta dentro de las instituciones hospitalarias, concordando con Muñoz y colab (12), que informa que los pacientes que tienen enfermedades graves, sufren, son seres de extrema vulnerabilidad y necesitan de cuidado especial.

El $80 \%$ de la población en estudio percibió ausencia de interacción entre la enfermera y el ser cuidado a diferencia de Rivera,(9) que sostiene que el cuidado es la esencia de la práctica de enfermería, que moviliza a la persona a través de una relación de ayuda generando conocimiento, respeto y ayuda mutua, rescatando siempre la dignidad humana, a semejanza de Poblete (6) que expresa que en Chile esta visión biomédica de la atención en salud en la cual se realizan los cuidados de enfermería, es lo que ha llevado a las enfermeras a una sobrecarga de trabajo de tipo biomédico y a labores en forma mecanizada, determinando que su trabajo se torne "invisible" en los campos clínicos, a diferencia de Prieto que expresa que la Enfermera como el sujeto de cuidado son personas con características, derechos y valores que los hacen únicos e irrepetibles y en la relación entre ellos se establece un vínculo humano que facilita el intercambio mutuo de vivencias, experiencias, y conocimientos que orientan el cuidado de Enfermería acorde a las necesidades del sujeto de cuidado.

El $92.66 \%$ de las personas enfermas expresan que no existe repercusión social en concordancia con Murrain (8), que manifiesta que a pesar de ser el mayor grupo para dar cuidado no es visible su actividad y en concordancia con Almenares(7) que indica 
que el cuidado de enfermería es una actividad profundamente humana que va más allá de la atención a la persona enferma El cuidado puede llegar a ser trascendente, profundamente espiritual e integrador para aquellos pacientes que sufren, a semejanza de Waldow, (4) que indica que los cuidadores deben estar preparados para ofrecer un cuidado que les proporcione confort, no solo de orden físico, sino también espiritual, utilizar técnicas de relajamiento, evidenciando y trasmitiendo, tanto por gestos, miradas $y$ palabras como en el silencio, coraje, solicitud y compasión y en concordancia con Poblete (6) quien expresa que en los servicios sanitarios brasileños no hay reconocimiento de la labor de la enfermera, lo que está llevando a la insatisfacción profesional de las enfermeras/os, en que observan la desvalorización de su trabajo en los campos clínicos y el alejamiento de la humanización de los cuidados, de la sociedad misma.

\section{CONCLUSIONES}

1. El $95 \%$ de enfermeras expresan que la enseñanza se basó en actividades procedimentales, con aprendizaje repetitivo.

2. En el $98 \%$ de enfermeras no tuvieron información sobre la ciencia de enfermería ni el soporte teórico.

3. El $82 \%$ de enfermeras refieren desconocimiento de aspectos centrales del cuidado enfermero.

4. El $80 \%$ de los enfermos expresan que sus experiencias sobre el cuidado enfermero fue negativa.

5. La prueba estadística de chi cuadrado establece que existe relación entre la trascendencia de la enseñanza del cuidado enfermero con la repercusión social.

\section{RECOMENDACIONES}

El desarrollo de las jornadas de capacitación para los enfermeros sobre el cuidado enfermero y la coordinación con las entidades formadoras para el fortalecimiento de los currículos de estudios.

\section{Correspondencia}

Dra. Sonia Olinda Velásquez Rondón

Correo electrónico: sovelasquez1@gmail.com

\section{BIBLIOGRAFIA}

\section{Kerouac S. Pensamiento Enfermero} España 1996

2. Meleis, Afaf. La relación entre la teoría, la práctica y la investigación con la calidad del cuidado de enfermería. Ponencia presentada en el VII Coloquio Panamericano de Investigación en Enfermería, Memorias Acofaen, Bogotá Noviembre de 2000, pp. 86 - 94.

3. Medina, J. Paz,M. La complejidad del cuidado y el cuidado de la complejidad: un tránsito pedagógico de los reduccionismos fragmentantes a las lógicas no lineales de la complejidad. Rev C Scielo 2006.

4. Waldow, V. La Vulnerabilidad del Ser Enfermo y su Dimensión de Trascendencia: Brasil. 2013

5. Báez V, y colab. El significado de cuidado en la práctica Profesional de enfermería 2009 Colombia.

6. Poblete M. Valenzuela S. Cuidado humanizado: un desafío para las enfermeras en los servicios hospitalarios Chile 2007.

7. Almenares C. Cuidado que trasciende más allá de la muerte. Colombia 2009.

8. Murrain E. Trascender en el Cuidado de Enfermería, una Oportunidad desde la Aplicación de la Teoría del Cuidado Cultural: Algunas Reflexiones, Colombia 2009.

9. Rivera, L. Triana, A. Cuidado humanizado de enfermería: Visibilizando la teoría y la investigación en la práctica clínica del Country Colombia, 2007.

10. Bernal, M Ponce G Propuesta para la enseñanza del cuidado en Enfermería, México Rev Universitaria ENEO UNAM.

2009. 
11. Jiménez M, Cárdenas L, Bardallo M, Do M, Martínez B. Desarrollo del pensamiento reflexivo y crítico en los estudiantes de enfermería de Iberoamérica. Rev. Investig. Cualitat. en Educación 2015. Vol 2(1). 2015

12. Muñoz Y y colab. Significado del Cuidado Humanizado en Egresadas de la Facultad de Enfermería Colombia 2007
13. Prieto G. Humanización del cuidado de Enfermería Colombia 2007.

Recibido:12/02/2016

Aprobado para Publicación:20/05/2016 